\title{
Spin Valve Effect and Hall Resistance in a Wide Parabolic Well
}

\author{
C. A. Duarte, G. M. Gusev, A. A. Quivy, and T. E. Lamas \\ Instituto de Física da Universidade de São Paulo, CP 66318, CEP 05315-970, São Paulo, SP, Brazil
}

Received on 4 April, 2005

\begin{abstract}
We report observation of the Hall slope change in wide $A l_{c} G a_{1-c} A s$ parabolic wells in the presence of a quasi-parallel magnetic field. Above the critical magnetic field $B>4 T$, the Hall resistance becomes temperature dependent and can be described by equation $R_{x y} / \cos \Theta \sim\left(B-B_{0}\right) / e n_{s}$, where $n_{s}$ is the electron density, $B_{0}=2$ 2.6 $\mathrm{T}$ and $\Theta$ is the angle between magnetic field and the normal to the well plane. The effect strongly depends on the electron density; it is observed only in parabolic wells, which are almost completely filled by electrons. We attribute the Hall slope change to the unusual behaviour of the effective $g$ factor in such parabolic well, which depends on the $A l$ composition and changes the sign along the well width.
\end{abstract}

Keywords: Spin valve; Hall resistance; $A l_{c} G a_{1-c} A s$ parabolic wells

\section{INTRODUCTION}

The Hall effect is very known and widely observed phenomenon in solid state physics. It arises from the Lorentz force that acts on a moving charge. The Hall resistance $R_{x y}$ is described by the conventional equation

$$
R_{x y}=-B / e n_{s}
$$

and offers a useful measure of the concentration $n_{s}$ of the charge carriers in the sample. In two-dimensional system in the strong magnetic field and low temperature the Hall resistance exhibits a plateaux, which equals to $h / e^{2}$ divided by an integer [1]. Another type of the Hall effect is an anomalous or extraordinary Hall effect discovered in ferromagnets almost 50 years ago [2]. It was found that the Hall resistivity in ferromagnets is larger than in nonmagnetic metals and can be fitted empirically by the formula $R_{x y}=R_{0} B+R_{S} M$, where $\mathrm{B}$ is applied magnetic field, $R_{0}$ is the ordinary Hall coefficient, and $R_{S}$ is the anomalous Hall coefficient.

In the present work we report observation of the anomalous Hall slope change in wide parabolic wells in quasi-parallel magnetic field. For tilt angle $\Theta \leq 90^{\circ}$ and low temperature we found that the Hall resistance at $B>4 T$ increases its slope twice and becomes temperature dependent. The ordinary Hall effect recovers at high $(T=50 \mathrm{~K})$ temperature and perpendicular magnetic field.

\section{EXPERIMENTAL RESULTS AND DISCUSSIONS}

The samples were made from $A l_{c} G a_{1-c} A s$ parabolic quantum well grown by molecular beam epitaxy. It included a 500, 750, 1000, 1500, 1700 and $2200 \AA$-wide parabolic $A l_{c} G a_{1-c} A s$ wells with $\mathrm{Al}$ content varying between 0 and 0.29 , bounded by undoped $A l_{c} G a_{1-{ }_{c}} A s$ spacer layers with $\delta-S i$ doping on two sides [3]. The mobility of the electron gas in our samples was $\sim(100-250) \times 10^{3} \mathrm{~cm}^{2} / \mathrm{Vs}$ and density $-n_{s}=(2-4) \times 10^{11} \mathrm{~cm}^{2}$, therefore our quantum wells were partially full with 2-5 subbands occupied. Four -terminal resistance $R_{x x}$ and Hall $R_{x y}$ measurements were made down to $1.5 \mathrm{~K}$ in a magnetic field up to $12 \mathrm{~T}$. We rotate sample in situ, so that magnetic field could be tilted with respect to the sample $(\mathrm{x}-\mathrm{y})$ plane in $\mathrm{x}$ or $\mathrm{y}$ directions. We denote the angle between $\mathrm{B}$ and the normal to the sample plane by $\Theta$. The characteristic bulk density is given by equation $n_{+}=\frac{\Omega_{0}^{2} m^{*} \varepsilon}{4 \pi e^{2}}$. The effective thickness of the electronic slab can be obtained from equation $W_{e f f}=n_{s} / n_{+}$. For partially filled quantum well $W_{e}$ is smaller than the geometrical width of the well $W$. The mobility of the electron gas in our samples was $590 \times 10^{3} \mathrm{~cm}^{2} / \mathrm{Vs}$ for narrow wells and $\sim 200 \times 10^{3} \mathrm{~cm}^{2} / V s$ for wider parabolic wells. We varied the electron sheet density by illumination with a red light-emitting diode. The ratio between the effective thickness of the electronic slab and geometric width $f=W_{\text {eff }} / W$ was less than 0.3 for $W<1000 \AA$ parabolic wells. After illumination the effective thickness increases, however, the electron density is saturated, and quantum well still remains partially occupied. The energy spectrum of the full PQW is more similar to the square quantum well than to a harmonic potential.

Application of the strong perpendicular magnetic field leads to the Zeeman splitting of the Landau levels, which is proportional to the average $g$-factor in parabolic well. The effective g-factor in $A l_{c} G a_{1-c} A s$ structures changes with composition [4]: $g(c)=-g_{0}+g_{1} c$, where $g_{0}=0.44$, and $g_{1}=2.7$. Therefore, g-factor increases monotonically from $g=-0.44$ (middle of the well) to $g=+0.4$ at the edge of the well $(c=0.3)$, and changes the sign at $c=0.13$. Fig. 1 shows the variation of the $g$-factor in this parabolic well along $z$ axis.

In perpendicular magnetic field g-factor should be calculated by averaging local $\mathrm{g}$-factor along $\mathrm{z}$-axis :

$$
<g>=\frac{1}{W} \int_{-W / 2}^{W / 2} g(z)|\Psi(z)|^{2} d z,
$$

where $\Psi$ is the electron wave function. In the presence of the in-plane magnetic field, however, evolution of 2D to 3D energy spectrum is expected, because magnetic length becomes smaller than the sample width. In this case the states in the different parts of the well along $\mathrm{z}$ axis has the different spin polarization: the center of the well is almost antialigned spin and the edge of the well is almost all aligned spins ( see Fig.1a). It may lead to the suppression of the electron motion in $z$ direction in crossed electric and magnetic fields, since the motion in $\mathrm{z}$ direction now requires the spin flip process, and, consequently to increase of the slope of the Hall effect in our case. Such effect is similar to the famous spin valve effect, which 
leads to the giant magnetoresistance in multilayers and has already found important applications [5].
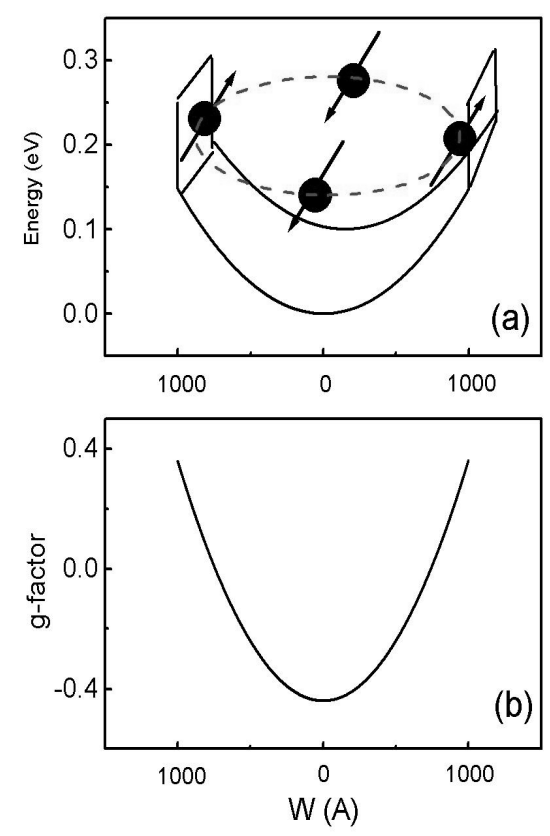

FIG. 1. Schematic view of the spin orientation of electrons in parabolic well in the presence of the strong in-plane magnetic field (a) and effective g-factor variation along $z$ (b).

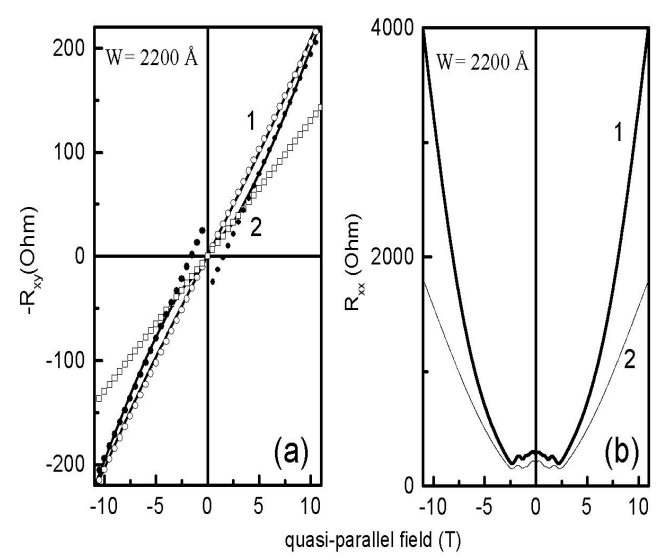

FIG. 2. The Hall(a) and diagonal (b) resistance of $2200 \AA$ wide parabolic well in quasi-parallel $\left(\Theta \approx 89.4^{0}\right)$ magnetic fields, $\mathrm{T}=4.2$ $\mathrm{K}$. The curves (1) and (2) are measured before and after illumination. Open circles and squares show the equation 1, full circles show the equation 3.

Figure 2 shows longitudinal $R_{x x}$ and Hall $R_{x y}$ resistances of $2200 \AA$-wide parabolic $A l_{c} G a_{1-c} A s$ well at $\Theta \approx 89^{\circ}$ as a function of applied magnetic field before and after illumination. The magnetic field is directed along the current flow. Note that for this tilt angle magnetic field is almost parallel to the sample plane. We keep small perpendicular component for Hall effect measurements. Indeed for completely parallel magnetic field no Hall effect is observed. We may see that the magnetoresistance reveals oscillations, sometimes called diamagnetic Shubnikov de-Haas $(\mathrm{SdH})$ oscillations, which results from the combined effect of the electric and magnetic fields. In quantum well with several subbands occupied such oscillations are interpreted as magnetic depopulation of the two-dimensional levels.

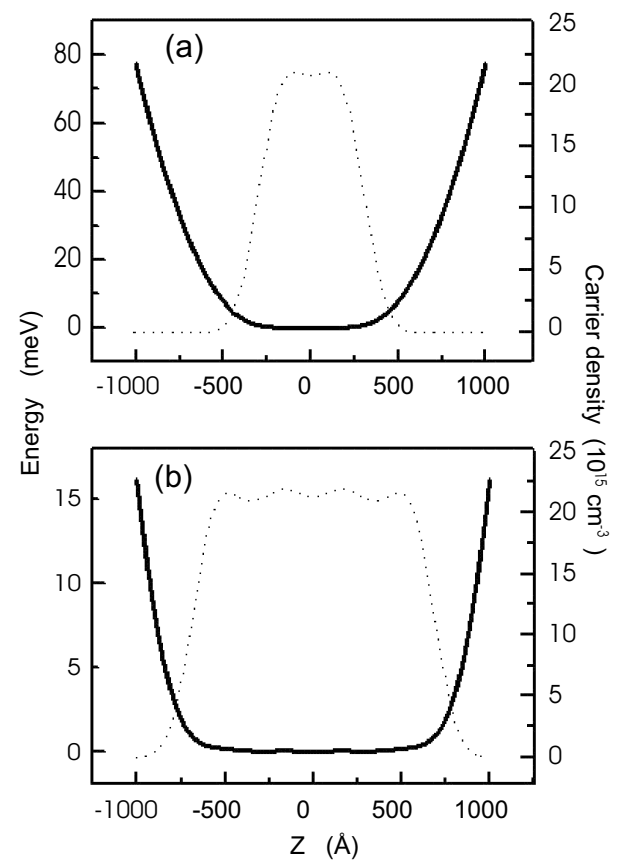

FIG. 3. Calculated total potential and electron density in $2200 \AA$ parabolic well for two different densities $n_{S}\left(10^{11} \mathrm{~cm}^{-2}\right): 1.3(\mathrm{a}), 3(\mathrm{~b})$.

Figure 2 shows that $R_{x x}$ exhibits 3 oscillations, therefore 3 subbands are depopulated in this $2200 \AA$-wide parabolic well. Before illuminations the Hall resistance is linear and can be described by equation 1 . After illumination the behaviour of $R_{x y}$ is dramatically changed: the Hall resistance demonstrates linear dependence on the magnetic field at low B, and at higher field, $R_{x y}$ deviates from the former linear dependence, and its slope increases. Such anomalous behaviour at high field is described by equation

$$
R_{x y} / \cos \Theta=-A \times\left(B-B_{0}\right) / e n_{s}
$$

where the value of $B_{0}$ depends on the sample width. We found that the coefficient $A$ strongly depends on the temperature: it gradually increases, when temperature decreases, and becomes 2 times larger at $\mathrm{T}=1.5 \mathrm{~K}$ than at low field and high temperatures. In perpendicular magnetic field we found ordinary quantum Hall effect.

We attribute such large Hall slope to the spin valve effect in $z$ direction, which also suppresses the motion in $y$-direction. Firstly, the Hall effect deviates from the ordinary slope at $B>4 T$, when magnetic length becomes smaller than the well width, and local $g$-factor turns to be $z$-dependent. In lower field $g$-factor should be calculated by averaging local $g$-factor 
along $z$-axis, given by equation 2 . Secondly, the Hall slope change is observed in quasi-parallel magnetic field, since the strong in-plane field makes local $g$-factor variable along $z$ axis. The change in the Hall slope also occurs in the presence of multiple carrier types as the subbands are depopulated, however this change has opposite sign and can not explain our observation in quasi-parallel magnetic field. Finally, the Hall slope change is observed only in parabolic well, which is almost completely filled by electrons. In these samples the effective g-factor changes the sign across the well, which can lead to the spin-valve effect. In partially filled parabolic well the sign change of the g-factor along the $\mathrm{z}$-direction does not occur, and Hall resistance is not affected by spin dependent transport. Fig. 3 shows charge distribution in parabolic quantum well before and after illumination. We see that the effective width of the electronic slab increases after illumination.

We also can vary the electron density by applying the voltage between the gate on the top of the sample and electron sheet.

Figure 4 shows the dependence of the diagonal and Hall resistance on the magnetic field for different gate voltages. We may see that the electron density decreases with negative gate voltage. Fig.5 demonstrates the behaviour of $R_{x x}$ and $R_{x y}$ in quasi-parallel magnetic field for different gate voltages.

The insert shows the ratio between the slope of the Hall resistance at low and high magnetic field as a function of the electron density. We may see that this ratio decreases with electron density decrease in accordance with our observation of the Hall slope change with illumination. However it is worth noting that the electric field in addition with depletion of the electron system may introduce the asymmetry and the shift of the wave function from the center of the parabolic well. We expect that the spin-valve effect will be larger for this experiments in comparison with the symmetric case.

We attribute the electron sheet density dependence or dependence of the Hall slope change on the width of the electronic slab to the spin valve effect in parabolic well. The magnitude of the Zeeman splitting in GaAs is $-0.064 \mathrm{meV}$ at $\mathrm{B}=2.5$ $\mathrm{T}$ for electrons in the center of the sample. At the edge of the well $\mathrm{g}$-factor is positive and the Zeeman energy has almost the same value, but opposite in the sign. Thus the spin flip process requires the energy $0.12 \mathrm{meV}$, which is comparable with temperature $\mathrm{T}=1.6 \mathrm{~K}$, and therefore may lead to the significant spin-valve effect. For partially occupied parabolic wells with $f=W_{\text {eff }} / W<0.3$ g-factor is varied between -0.2 and -0.44 , and does not change the sign. Indeed the spin-valve effect is not expected in this case, in accordance with our observation.

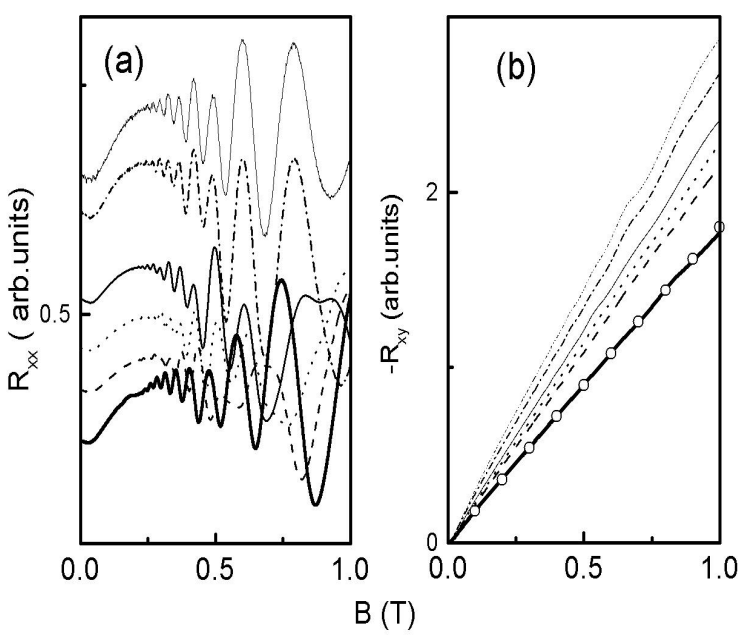

FIG. 4. The longitudinal and Hall resistance of the $2200 \AA$ wide parabolic well as a function of magnetic field at $\Theta=0^{0}$ for different gate voltages $V_{g}$ (Volts): 0 (thick line),2(dashes),4(dots),6(thin line),8(dashes-dots), 10 (dots-dots-dashes).

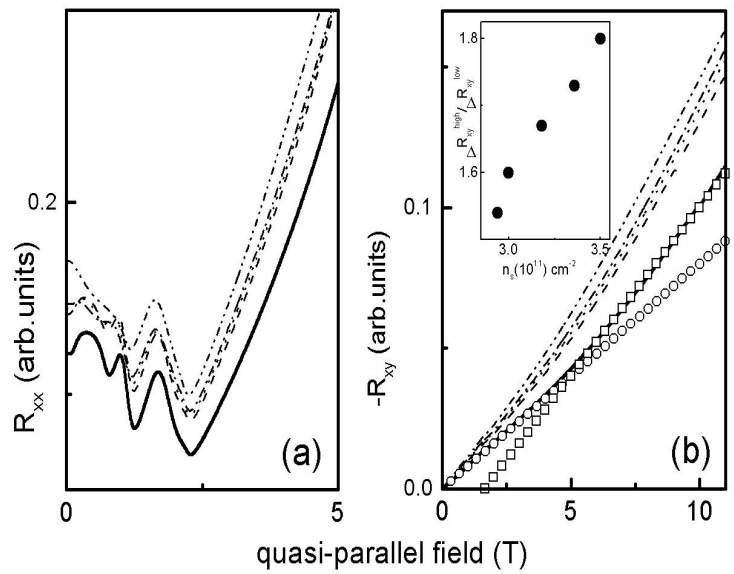

FIG. 5. The longitudinal and Hall resistance of the $2200 \AA$ wide parabolic well as a function of magnetic field at $\Theta \approx 89.6^{0}$ for different gate voltages $V_{g}$ (Volts): 0(thick line),1(dashes),2(dots),4(thin line).Open circles show the equation 1, squares show the equation 3 . The insert shows the ratio between the slope of the Hall resistance at low and high magnetic field

\section{CONCLUSION}

We demonstrated that the variation of the $g$-factor along the well width is responsible for the Hall slope change in wide parabolic well in the presence of the strong in-plane magnetic field. We attribute such large Hall slope to the spin valve effect in $z$ direction. Since in the tilted field the electronic motion in the $z$-direction and $y$-direction are strongly coupled, the transversal component of the conductance is suppressed, and Hall resistance grows. 
Acknowledgments

Support of this work by FAPESP, CNPq (Brazilian agencies)is acknowledged.
[1] The quantum Hall effect, edited by R. E. Prange, S. M. Girvin, New York, 1990

[2] C. M. Hurd, The Hall Effect in Metals and Alloys, Plenum Press, New York, 1972.

[3] G. M. Gusev, A. A. Quivy, T. E. Lamas, J. R. Leite, A. K. Bakarov, A. I. Toropov, O. Estibals, J. C. Portal, Phys. Rev. B,
65, 205316 (2002).

[4] C. Weisbuch, C. Hermann, Phys. Rev. B, 15, 816 (1977).

[5] G. A. Prinz, Science 282, 1660 (1998); A. Wolf et al., ibid. 294, $1488(2001)$ 